\title{
Stimulated Raman scattering in the evanescent field of liquid immersed tapered nanofibers
}

\author{
Liye Shan, ${ }^{1}$ Gilles Pauliat, ${ }^{1}$ Guillaume Vienne, ${ }^{2, a)}$ Limin Tong, ${ }^{2}$ and Sylvie Lebrun ${ }^{1, b)}$ \\ ${ }^{1}$ Laboratoire Charles Fabry, Institut d'Optique, CNRS, Univ Paris-Sud, 2 av. A. Fresnel, \\ 91127 Palaiseau cedex, France \\ ${ }^{2}$ State Key Lab of Modern Optical Instrumentation, Department of Optical Engineering, \\ Zhejiang University, Hangzhou 310027, China
}

(Received 22 February 2013; accepted 4 May 2013; published online 22 May 2013)

\begin{abstract}
We present the first experimental demonstrations of stimulated Raman scattering in a liquid probed by the evanescent field of a tapered silica nanofiber. Raman scattering by a pure liquid, ethanol, or mixture of liquids, toluene diluted in ethanol, is investigated. Given the large choice of available materials for the medium surrounding the nanofiber, these demonstrations pave the way to the exploration of a new class of experiments and devices. (C) 2013 AIP Publishing LLC. [http://dx.doi.org/10.1063/1.4807170]
\end{abstract}

Tapered nanofibers are optical fibers stretched until their diameter becomes comparable to the optical wavelength. ${ }^{1,2}$ At these small diameters, the guided light exhibits a pronounced evanescent field that extends outside the fiber and senses the surrounding medium. Significant optical nonlinearities, also called "evanescent nonlinearities," could thus be excited in this surrounding medium. ${ }^{3} \mathrm{We}$ are interested in the Raman evanescent nonlinearity occurring in a liquid in which the nanofiber is immersed. A pump beam injected as a guided mode at one end of the nanofiber experiences spontaneous Raman scattering: the pump photons are spontaneously scattered into photons of lower energy, the Stokes photons. ${ }^{4}$ Because part of these Stokes photons are scattered in the guided mode of the nanofiber, they can be subsequently amplified at the expense of the pump beam by stimulated Raman scattering. The pump guided mode is thus progressively attenuated along the fiber in favor of the growth of a Stokes guided mode. As a nanofiber is endlessly single mode, a Raman cascade can be observed: if the Raman gain is large enough, this first Stokes guided mode can itself give rise to a second Stokes order, and so on. The frequency shift between the pump and first Stokes waves (or first and second Stokes orders) is a characteristic of the chosen liquid. For a given pump wavelength, the converted Stokes wavelength can thus be selected by a correct choice of the liquid.

Using such "evanescent nonlinearities" to excite the Raman nonlinear effect in a liquid in which the nanofiber is immersed was recently investigated from a theoretical point of view. ${ }^{5}$ In this recent paper, the main features of these Raman evanescent nonlinearities were predicted:

- Large modal Raman gains can be reached. Typically with a pump beam at $532 \mathrm{~nm}$, modal Raman gains, $g_{S 1}$, up to a few $\mathrm{m}^{-1} \cdot \mathrm{W}^{-1}$ are expected for sub-micrometer glass nanofibers immersed in common liquids such as ethanol, toluene, and benzene. ${ }^{5}$

\footnotetext{
${ }^{a)}$ Present address: Data Storage Institute, Agency for Science, Technology and Research, (A*STAR), Singapore 117608

${ }^{\text {b)} E l e c t r o n i c ~ m a i l: ~ s y l v i e . l e b r u n @ i n s t i t u t o p t i q u e . f r . ~}$
}

- The critical parameter for sub-nanosecond green pulses is about $\gamma=g_{S 1} P_{\text {crit }} L \approx 23$, with $L$ the nanofiber length; the critical power $P_{\text {crit }}$ being the power for which the output pump power equals the output Stokes power.

- Although Raman excitation only occurs in the liquid surrounding the nanofiber, the Stokes wave is produced under the form of a $\mathrm{HE}_{11}$ mode, thus with its maximum in its center. It thus can be efficiently coupled back in a standard fiber.

The large modal Raman gain combined with the moderate value of the critical parameter is interesting as it allows performing the evanescent Raman conversion with subnanosecond pulses whose peak power is a few hundred watts only for a nanofiber length of about $L \approx 10 \mathrm{~cm}$.

The purpose of this communication is to experimentally evidence such an evanescent Raman conversion. We first provide a brief description of the pulling platform we specifically designed to draw nanofibers whose characteristics were defined previously. ${ }^{5}$ We then report on two evanescent Raman field experiments, the first with ethanol as the active medium, the second with toluene.

The most stringent nanofiber requirements are for the experiment conducted with ethanol, as it possesses a relatively low Raman coefficient. At the operating wavelength of $532 \mathrm{~nm}$, the maximum modal Raman gain for a nanofiber immersed in ethanol is expected to be $g_{S 1} \approx 1.04 \mathrm{~m}^{-1} . \mathrm{W}^{-1}$ for the optimum radius of $215 \mathrm{~nm}$. The critical parameter is about $\gamma=g_{S 1} P_{\text {crit }} L \approx 23$ as defined by our previous modeling. ${ }^{5}$ In order to observe the evanescent Raman effect, the nanofiber length $L$ should be large enough to keep the critical power $P_{\text {crit }}$ below the laser damage threshold of silica. This laser-induced damage threshold in the sub-nanosecond regime we use is relatively low. ${ }^{6}$ In the sub-nanosecond regime and with a low repetition rate (here $500 \mathrm{~Hz}$ ), damage is initiated by a linear absorption and thus only depends on the total energy deposited by a single pulse. This energy threshold is

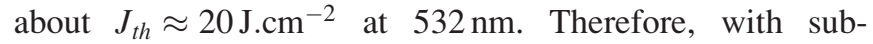
micrometer nanofibers, the critical power required to observe the Raman evanescent effect should be kept below a few hundred watts to avoid damaging the nanofiber. Considering 


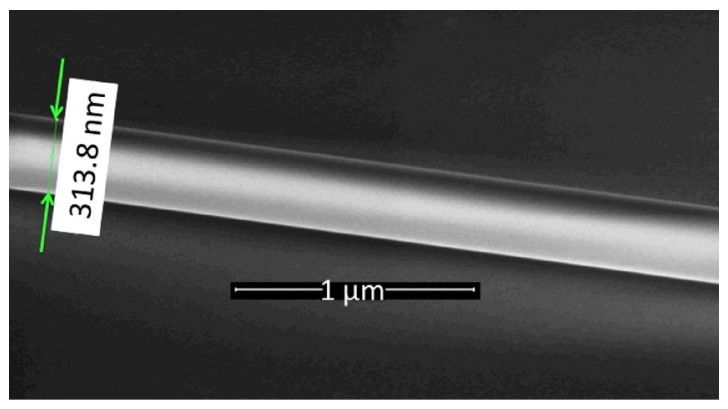

FIG. 1. Scanning electron microscope image of a nanofiber whose diameter is $314 \mathrm{~nm}$.

this constraint together with the critical parameter, the required nanofiber should be at least a few centimeters in length.

The fiber to be pulled (a standard SMF-28 telecommunication fiber) is attached at two computer-controlled translation stages. These two translation stages elongate the fiber to create the nanofiber while a heater softens its central part. ${ }^{7,8}$ The nanofiber shape is fully controlled by the trajectories of the two stages. After pulling, the un-tapered parts, upstream and downstream the nanofiber, allow a very easy light injection and collection. With this system, we routinely achieved light transmission larger than $90 \%$ over the full fiber, even with nanofiber diameters of a few hundred nanometers. A scanning electron microscope image of the smallest part of such a fiber is illustrated in Figure 1.

The full scheme of the experiment is depicted in Figure 2. The $1.06 \mu \mathrm{m}$ light from the pulsed microlaser is first frequency doubled with the KTP crystal. It operates at $500 \mathrm{~Hz}$. At $532 \mathrm{~nm}$, the full width at half maximum of the pulses is 510 ps. The remaining infrared light is then filtered out. A half-wave plate followed by a polarizing beam splitter cube allows controlling the power injected in the SMF-28 fiber. At $532 \mathrm{~nm}$, the SMF-28 supports several transverse modes. In order to precisely determine the light intensity inside the single mode nanofiber, we have to ensure that the light reaching the nanofiber is under the form of the $\mathrm{HE}_{11}$ mode only. Therefore, to reject all the higher transverse modes injected inside the SMF-28, we first create a single-mode filter upstream the nanofiber by stretching the SMF-28 down to a diameter of $40 \mu \mathrm{m}$ over a length of $1 \mathrm{~cm}$. This tapered section is embedded in an optical adhesive whose refractive index is higher than that of silica. Such a filter only transmits the fundamental $\mathrm{HE}_{11}$ mode. ${ }^{9}$ The fiber length between this filter and the nanofiber part is smaller than $20 \mathrm{~cm}$. Over these $20 \mathrm{~cm}$, bending is minimized to be sure that this fundamental

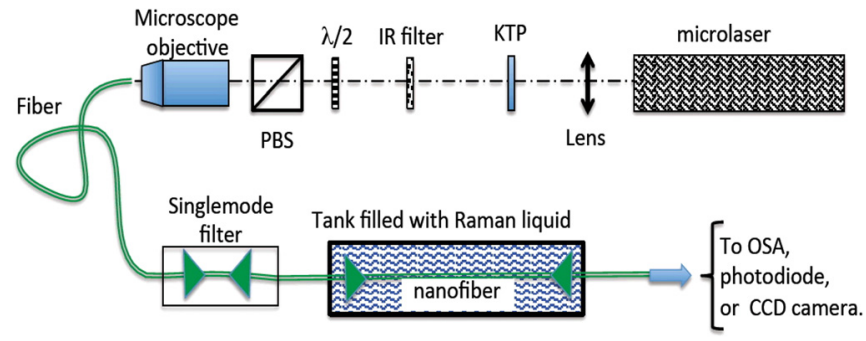

FIG. 2. Scheme of the experimental set-up used to test Raman evanescent nonlinearities. PBS is a polarizing beam splitter; the green triangles on the fiber represent the taper sections of the fiber. mode propagates without being coupled to higher order modes. We checked this point by controlling the transmitted power during the pulling process. This transmission never falls below $90 \%$ indicating that more than $90 \%$ of the power propagates under this fundamental mode. After the pulling process, the nanofiber is immediately immersed inside a tank filled with the Raman liquid. This tank is longer than the nanofiber to ensure that the nanofiber radius at locations where the nanofiber plunges in and emerges from the liquid is larger than a few micrometers. For such radii, the $\mathrm{HE}_{11}$ mode does not have any significant evanescent field: the entrance and exit in the liquid are performed with negligible loss.

For analyzing the Raman effect, we let the beam from the fiber either propagate in free space for capturing images with a CCD camera, or we sent it through a multimode fiber (to minimize any further non-linear effects) to an Optical Spectrum Analyzer, OSA.

As a preliminary test, before pulling the nanofiber, we increased the injected power to check that, in our intensity range, no by-side nonlinear effect appears in the silica fiber. After pulling the nanofiber, but before immersing it in the liquid, we observed the appearance of supercontinua for nanofiber diameters ranging approximately between 0.5 and $1 \mu \mathrm{m}$. These observations are in agreement with results previously reported. ${ }^{9}$ These supercontinua disappeared upon immersion in the liquid. This was expected as the immersion inside the liquid considerably increases the absolute value of the group velocity dispersion: the phase matching condition at the origin of the creation of supercontinua is no more fulfilled.

The first experiment is conducted by immersing the nanofiber in a pure liquid, ethanol. We selected ethanol, as it is a non-toxic liquid with relatively well-known refractive index and Raman coefficient. Furthermore, an experiment achieved with this liquid whose Raman gain is moderate gives us confidence in the successful evanescent Raman conversion with other liquids. The targeted nanofiber diameter is $440 \mathrm{~nm}$ over a length of $6 \mathrm{~cm}$. Once the nanofiber is immersed in ethanol, we increase the input power step by step.

As a first observation, we let the beam from the fiber propagate to a CCD camera in front of which we put a color filter at the Stokes wavelength $(630 \mathrm{~nm})$. Even if the Raman excitation appears only in the evanescent field surrounding the nanofiber, the output Raman Stokes wavefront presents a maximum in its center. This wave is single-mode as expected: slightly moving the final fiber section does not modify this profile.

A typical spectrum taken with the OSA is shown in Figure 3. It has been recorded with an injected pulse energy of $0.22 \mu \mathrm{J}$. Beside the peak corresponding to the transmitted pump at $532 \mathrm{~nm}$, the first Stokes order at $630 \mathrm{~nm}$ is clearly visible. On this spectrum, the lines are not resolved, as the OSA does not allow performing large wavelength scans at the highest resolution. With an additional measurement conducted over the Stokes line only at the largest available resolution, we measured a Stokes linewidth of $0.15 \mathrm{~nm}$ not taking into account the finite resolution of the Optical Spectrum Analyzer, $0.05 \mathrm{~nm}$. 


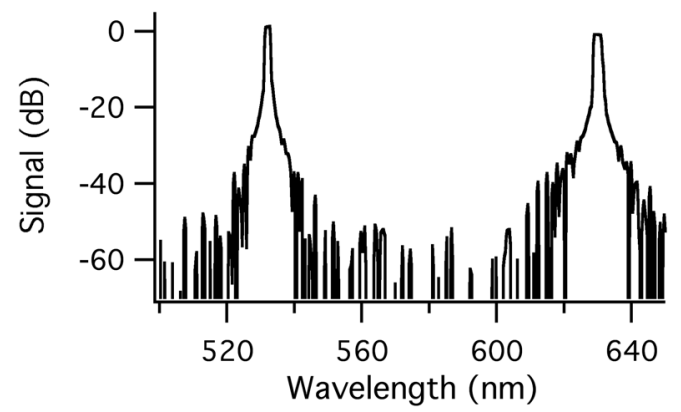

FIG. 3. Typical spectrum for a nanofiber immersed in ethanol: pump wavelength $532 \mathrm{~nm}$ and pulse energy $0.22 \mu \mathrm{J}$.

In Figure 4, we report the measured output pump and Stokes energies versus the input pump energy, as green squares and red disks, respectively.

Even well above threshold, a significant part of the pump energy remains in the output pump beam. Indeed the Raman interaction mainly depletes the pump pulse around its maximum, the aisles of the pulse remaining largely undepleted. This is confirmed by pulse width measurements. While the half-width at full maximum of the input pump pulse is $510 \mathrm{ps}$, we measured a half-width at full maximum of the Stokes pulse down to $120 \mathrm{ps}$.

We never observed the second Stokes order at $773 \mathrm{~nm}$. Indeed, because the modal Raman gain for this second Stokes order is more than two times lower than for the first Stokes order, it is expected to appear for pump energies about 5 times larger than for the appearance of the first Stokes. ${ }^{5}$ For such large energies, the pump induced optical damage burns the nanofiber.

The fact that the ratio of the modal Raman gain for the second Stokes to the modal Raman gain of the first Stokes is small is nevertheless adventitious. This ratio depends on the chosen liquid and on the nanofiber diameter. We indeed observed the second Stokes order for other liquids as illustrated for toluene below.

The second experiment is conducted with toluene as the Raman liquid. Its refractive index is higher than that of silica. In order to guide light, it should be diluted within a solvent of lower refractive index to make the refractive index of the mixture lower than the one of silica. We selected ethanol as the solvent. The volume fraction of toluene in the mixture is $50 \%$. Ethanol is inactive, its Raman coefficient being

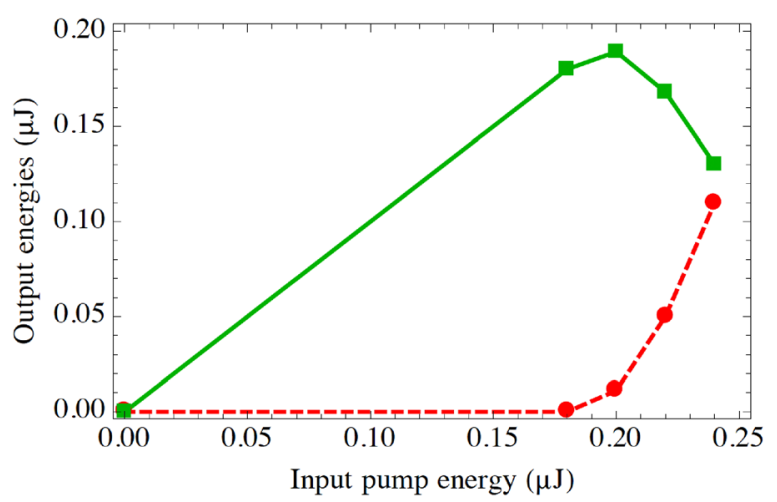

FIG. 4. The experimental measured pump and Stokes transmitted energies (green squares and red disks). The uncertainties on the intensities measurements are about $20 \%$. The two curves are just guides for the eye.

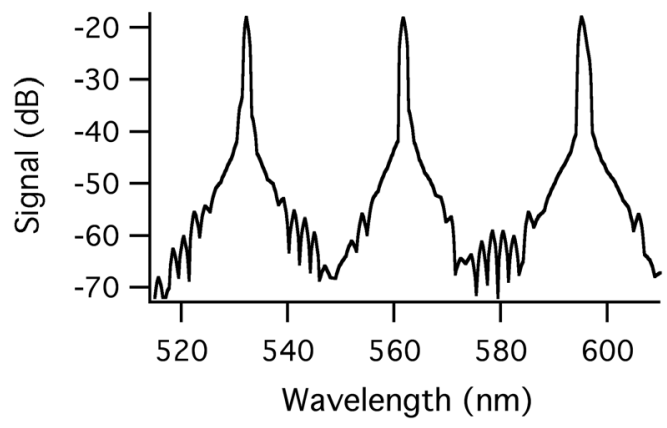

FIG. 5. Spectrum obtained with a nanofiber immersed in a mixture of toluene and ethanol (50/50). Beside the pump beam at $532 \mathrm{~nm}$, we observe the first Stokes order at $562 \mathrm{~nm}$ and second Stokes order at $596 \mathrm{~nm}$, which are strongly excited.

lower than the one of toluene. The targeted nanofiber diameter was $700 \mathrm{~nm}$ over a length of $6 \mathrm{~cm}$. From the pump beam at $532 \mathrm{~nm}$, the first and second Stokes orders at $562 \mathrm{~nm}$ and $596 \mathrm{~nm}$ are strongly excited. The spectrum reported in Figure 5 was recorded with a pump energy of $0.54 \mu \mathrm{J}$. Compared to the first experiment with pure ethanol, the higher Raman coefficients of toluene allow the excitation of a Raman cascade: the pump excites a first Stokes wave that itself gives rise to a second Stokes order.

In conclusion, we performed the first demonstrations of Raman conversion in the evanescent field of a tapered nanofiber. These experiments are in good agreement with our predictions. Even with the low cost sub-nanosecond microlaser we used, the Raman critical powers remain below the laser damage threshold of silica. Such experiments should be even simpler to conduct with shorter pulses. The laser damage threshold is indeed a function of the total energy in the pulse while the Raman effect depends on the intensity. Decreasing the pulse duration should thus enhance the intensity operating range of such devices: that is the intensity ranging from the Raman threshold up to the silica damage threshold. With shorter pulses, the second Stokes order should be easily excited even in ethanol.

It is worth mentioning that, as the active Raman medium, we deliberately selected ethanol in our first experiment although its Raman gain is not among the highest. Similar experiments should thus be achievable with most liquids or mixtures of liquids. As an alternative to liquids, sol-gel or polymers could be used for embedding the nanofiber. ${ }^{10,11}$ In addition to providing new possibilities for engineering the device, they protect the nanofiber and make it easier to handle. The exploitation of Raman and parametric "evanescent nonlinearities" with nanofibers is probably widening the field of applications of nanofibers as did the "evanescent nonlinearities" for silicon-organic hybrid systems. ${ }^{12}$

We thus believe that these first demonstrations open up the way to a full range of exciting new experiments using these "evanescent nonlinearities."

The authors thank Anne-Lise Coutrot from the Laboratoire Charles Fabry, Palaiseau, France, and Christophe Dupuis from the Laboratoire Photonique et Nanostructures, Marcoussis, France, for the measurements with the scanning electron microscope. 
${ }^{1}$ L. M. Tong, J. Lou, and E. Mazur, Opt. Express 12, 1025 (2004).

${ }^{2}$ L. M. Tong, F. Zi, X. Guo, and J. Lou, Opt. Commun. 285, 4641 (2012).

${ }^{3}$ M. A. Foster, A. C. Turner, M. Lipson, and A. L. Gaeta, Opt. Express 16, 1300 (2008).

${ }^{4}$ G. P. Agrawal, Nonlinear Fiber Optics (Elsevier, Amsterdam, 2010).

${ }^{5}$ L. Shan, G. Pauliat, G. Vienne, L. M. Tong, and S. Lebrun, J. Eur. Opt. Soc. Rapid Publ. 8, 13030 (2013).

${ }^{6}$ N. Kuzuu, K. Yoshida, H. Yoshida, T. Kamimura, and N. Kamisugi, Appl. Opt. 38, 2510 (1999).
${ }^{7}$ T. A. Birks and Y. W. Li, J. Lightwave Technol. 10, 432 (1992).

${ }^{8}$ C. Baker and M. Rochette, Opt. Mater. Express 1, 1065 (2011).

${ }^{9}$ S. Leon-Saval, T. Birks, W. Wadsworth, P. St. J. Russell, and M. Mason, Opt. Express 12, 2864-2869 (2004).

${ }^{10}$ L. Xiao, M. D. W. Grogan, W. J. Wadsworth, R. England, and T. A. Birks, Opt. Express 19, 764-769 (2011).

${ }^{11}$ N. Lou, R. Jha, J. L. Domínguez-Juárez, V. Finazzi, J. Villatoro, G. Badenes, and V. Pruneri, Opt. Lett. 35, 571-573 (2010).

${ }^{12}$ J. Leuthold, C. Koos, and W. Freude, Nature Photon. 4, 535-544 (2010). 